\title{
Thick DLC Films Deposited By PECVD on the Internal Surface of Cylindrical Substrates
}

\author{
D. Lusk, M. Gore
}

Sub-One Europe Ltd., 1 Berry Street, Aberdeen, Scotland, AB25 1HF

W. Boardman, T. Casserly, D. Upadhyaya, A. Tudhope

Sub-One Technology, 4464 Willow Rd., Bldg. 103, Pleasanton, CA, 94588

\begin{abstract}
A new and exciting technique for performing DLC deposition on the inside of cylindrical substrates, in particular pipes, will be described. Using the hollow cathode effect (HCE), a high density plasma can be generated within such cylindrical substrates by using Plasma Enhanced Chemical Vapor Deposition (PECVD). As the pipe itself is the vacuum chamber, such high density plasmas can be maintained by using asymmetric bipolar direct current (DC) pulsed power. Very high deposition rates can thus be achieved of the order of 1 micron/min. A hydrocarbon precursor $\left(\mathrm{C}_{2} \mathrm{H}_{2}\right)$ is used to deposit thick DLC films which are inert and have a high corrosion resistance. Adhesion to the metallic substrate is improved by adding silicon to the DLC layer. These films also have excellent erosion and wear resistant properties and the process can be optimized depending on what film properties are most vital for whatever application the coating is required for. Corrosion and wear resistance are also improved by having a pure DLC layer on top of the deposited structure. The actual process and deposition system will be described in detail as well as how the technology works and how such high density plasmas can be maintained for various lengths and diameters of pipe. Testing of such novel DLC films was done by various techniques and results will be shown of hardness, adhesion, layer thickness, wear and corrosion resistance. A vast number of applications can greatly benefit from this novel process, on both a large and small scale. Examples of such applications would be industrial piping, offshore drilling, chemical delivery systems, gun barrels and medical devices.
\end{abstract}

\section{INTRODUCTION}

Numerous deposition techniques for applying a protective coating to the inside of cylindrical substrates, such as pipes, have been studied elsewhere [1-5]. Most common techniques such as electroplating, painting or spraying (thermal, arc and high velocity Oxy-Fuel) are considered for this application. Typically these involve specialised materials such as tungsten carbide and most require further finishing of the coating surface to obtain a useful smoothness and finish. These traditional coating methods 
were primarily developed to coat exterior or exposed surfaces and often have limited effectiveness when applied to coating internal surfaces outside of a limited size range. In addition, many of the raw materials or process by products can be toxic or environmentally unfriendly.

This paper will present in detail, a unique process for depositing extremely hard, smooth, corrosion and erosion resistant films on the interior surface of metallic components such as pipes and tubes through a hollow cathode plasma immersion ion processing (HCPIIP) method. This technique utilises the hollow cathode effect (HCE) to generate and maintain an extremely high density plasma inside the part to be coated which can be used to form coatings and films through a plasma enhanced chemical vapour deposition (PECVD) process in the presence of certain precursor gases.

By using carbon containing precursors, such as acetylene, extremely hard diamond-like carbon (DLC) films can be formed on the inside of the component. As has been extensively reported elsewhere [6], DLC films have unique combinations of high hardness, low friction, electrical insulation, chemical inertness, biological compatibility, smoothness and resistance to wear. The unique properties of these films have attracted great interest in a large number of industries including oil and gas, automotive, aerospace, medical, military and paper. Most of the deposition techniques for producing DLC films are slow, $<<1 \mu \mathrm{m} / \mathrm{hr}$, have limited capability or effectiveness in coating internal or unexposed surfaces, and produce high residual stresses in the film so limiting the practical coating thickness to a few $\mu \mathrm{m}$ which has limited the rate of commercial adoption for these coatings. In the case of piping and tubing, which typically transmit a potentially corrosive chemical down their internal area, the attractive properties of DLC coatings can be used to reduce corrosion, wear and abrasion on the inside of the part to increase useful lifetimes. In addition, the inherent smoothness and low coefficient of friction properties of DLC films could be of benefit in reducing line losses as liquids and slurries flow through a coated tube.

An important advantage of the described PECVD process is the ability to introduce different precursor materials during the coating process and thus change the film composition or introduce multiple layer films during growth. This allows formation of adhesion layers at the coating interface to promote strong bonding of the film, or multiple layer structures to enhance specific properties of the film

This article will give an overview of the vacuum deposition system and the technology which goes behind the deposition of these films. A detailed analysis of the characterization and testing of the described DLC films will be given as well as an overview of the range of properties that can be achieved. The resulting DLC films are unique in that they are formed on interior surfaces, but more interestingly these films can be grown at rates approaching $1 \mu \mathrm{m} / \mathrm{min}$ to a total thickness of around $75 \mu \mathrm{m}$. The narrow dark space sheath intrinsic to hollow cathode discharges promotes outstanding conformal coverage making the process suitable for coating threads or other complex internal profiles. Finally some detailed information on potential applications will be reviewed to describe potential commercial benefits of the coating. 


\section{EXPERIMENTAL}

A novel hollow cathode plasma immersion ion processing (HCPIIP) deposition method has been developed with the intention of depositing hard thick DLC films on the inside of metallic components. This method uses the hollow cathode effect (HCE) to generate an extremely high density plasma within the pipe itself. Maintaining a hollow cathode discharge within the component is important for rapid growth of thick DLC based films. The critical parameters for maintaining such a discharge are the internal diameter of the part and the operating pressure.

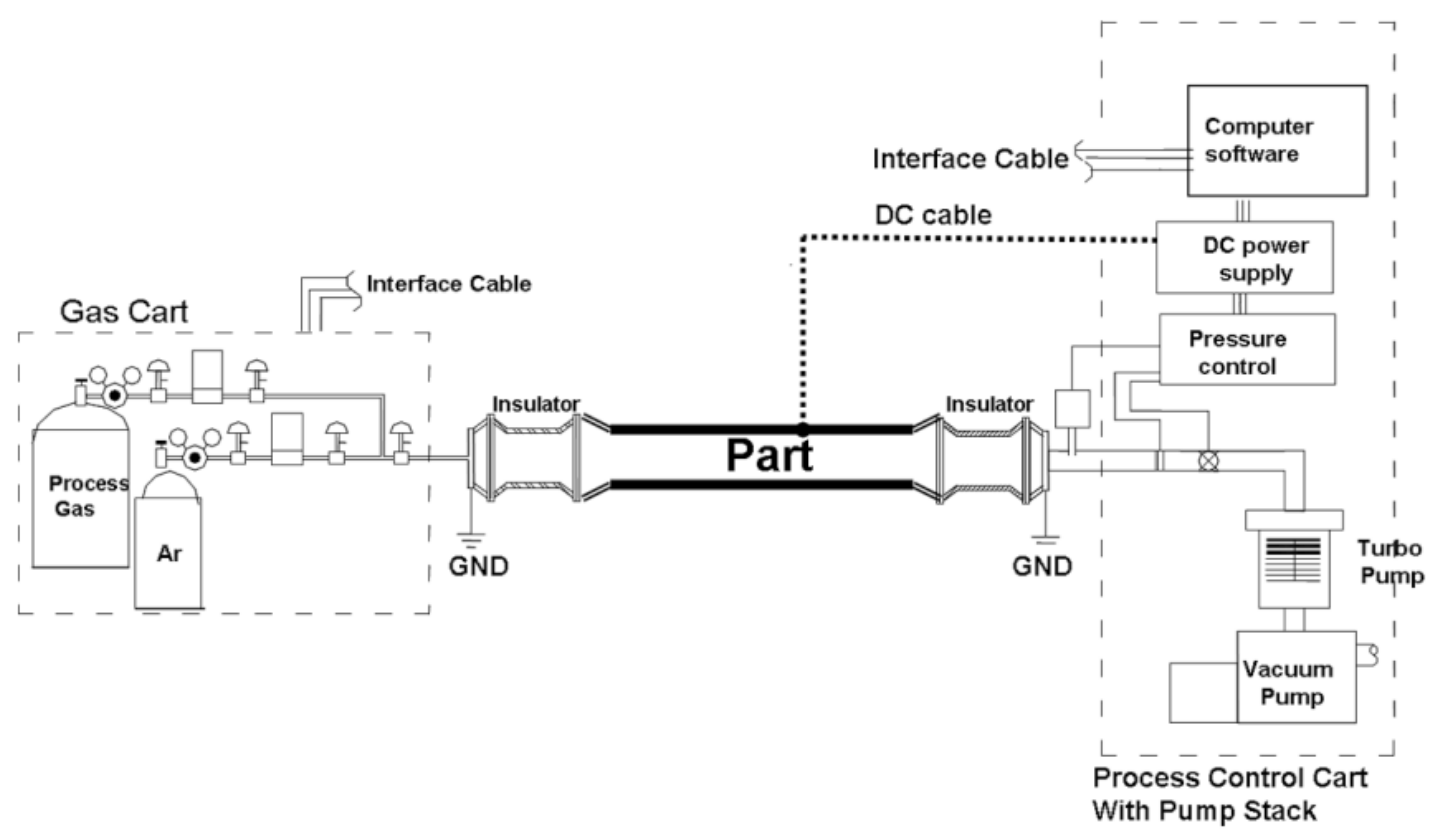

Figure 1 : Diagram of process set-up [7]

Figure 1 shows a schematic of the deposition system. From the main gas source system, process and precursor gases are introduced into the entrance head. The gas then travels down the pipe where it is ionized quickly and deposition takes place. At the exit head, a high vacuum pump and throttle valve are used to control operating pressure and pump away any by-products. The pipe or component is a critical element of the plasma electrical circuit, serving as the negatively biased cathode with respect to positively biased anodes, located at the entrance and exit heads. The anodes are electrically isolated from the cathode to be coated by insulating spacers. The cathode bias plays an important role in improving the stress, density and adhesion of the films by ion bombardment energy. Under suitable vacuum conditions, a bipolar asymmetric DC pulse is used to maintain a high density plasma inside the component. Energetic positive ion bombardment is controlled by the magnitude of the applied voltage and by the pressure. Gas flow and pumping speed are varied such that the pressure inside the component provides a regime where a hollow cathode discharge can be maintained under the applied component voltage. This pressure is such that the electron mean free path is adjusted to the pipe diameter, which cause high energy electrons to oscillate across the opposing cathode walls resulting in multiple ionizing collisions and a high density plasma being maintained [7]. As DLC films are insulative, short pulses (< $30 \mu$ secs) are used to dissipate any positive charge build up on the coating surface. This charge is compensated when the plasma sheath collapses during the off cycle. Adjusting the duty cycle of the DC waveform can allow good control of the film uniformity and thus allows the gas to replenish during the off cycle. A high density 
plasma promotes high deposition rates and also allows some influence over the residual stress state and bonding state of the applied film.

Film adhesion is promoted through use of a high bias voltage, which can result in ion implantation below the surface of the film, along with suitable precursors introduced at the start of a deposition cycle. Also, in-situ sputtering with argon and/or nitrogen can be used to clean and heat the component surface prior to coating.

This technique can be used to investigate a number of different aspect (length/diameter) ratios. High aspect ratio pipes can be coated with higher flows and low duty cycles [7]. Currently an aspect ratio of 24/1 is possible. Figure 2 shows examples of DLC coated SS parts of different aspect ratio [8].

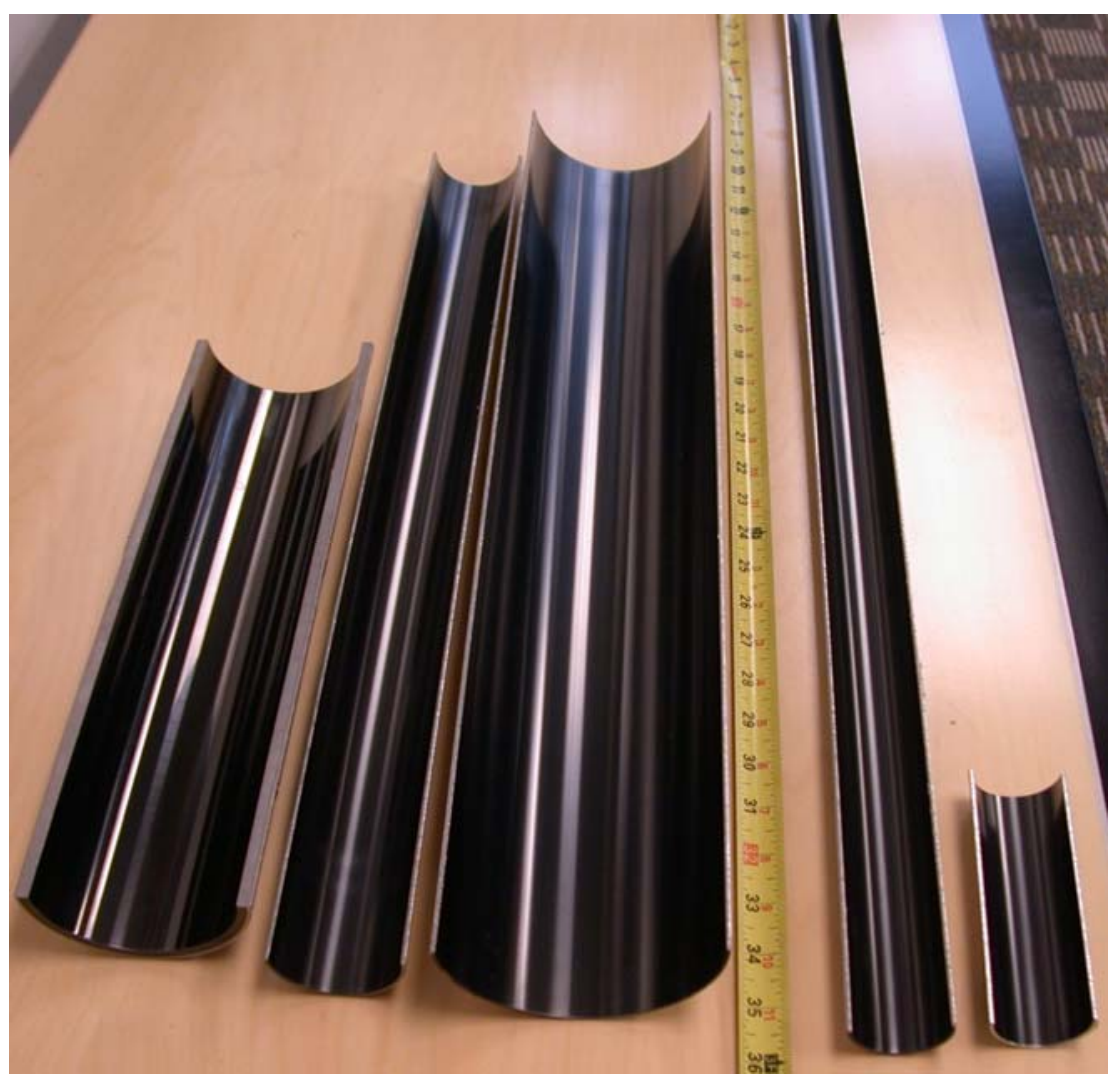

\section{Figure 2: DLC Coating on Internal Surfaces of Pipes of Various Aspect Ratios [8]}

Coating a range of different aspect ratio pipes places importance on controlling critical scaling parameters to maintain an operating regime suitable for the hollow cathode effect. Figures 3(a) and 3(b) show the pressure required to generate a hollow cathode plasma for large and small diameter tubes. The data shown in these figures was generated from Ref.9 (for 3(a)) and from Ref.10 (for 3(b)) using constant $~$ pressure $\mathrm{x}$ diameter. As can be seen, the data from the two references do not match exactly, however this is due to the fact that a hollow cathode discharge can be maintained over a wide pressure range for a given spacing, although it may lose plasma density. More details about the deposition process can be found in reference [7]. 
(a) large diameter tubes

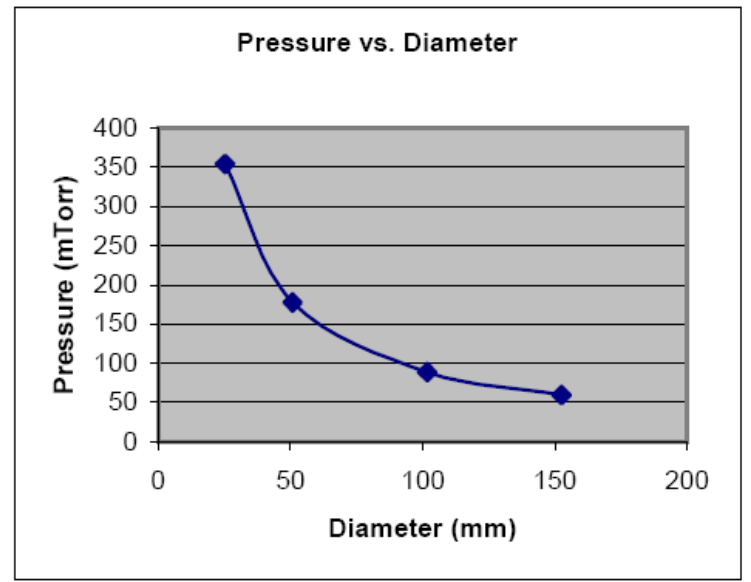

(b) small diameter tubes

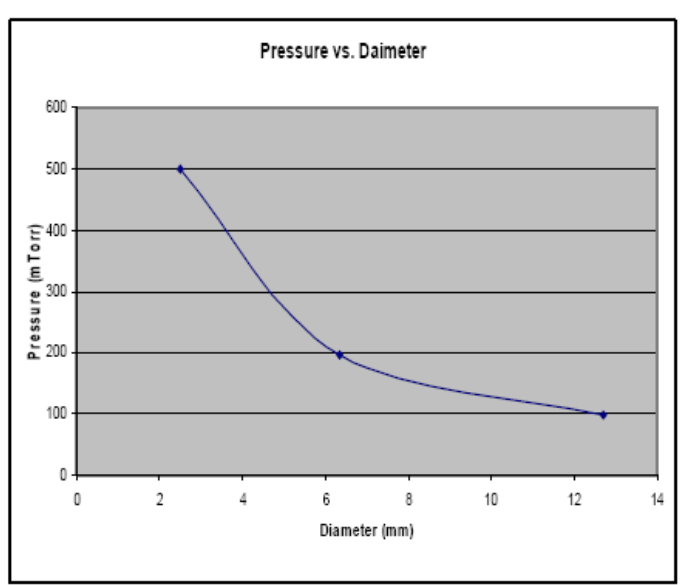

Figure 3: Pressure versus diameter for (a) large and (b) small diameter tubes $[9,10]$

One of the key aspects of this process is the pendulum motion of hot electrons between the cathode walls which helps to maintain the high density plasma. Also Langmuir probe measurements from a hollow cathode plasma have shown that an ion density of 2 $\mathrm{x} 10^{12} \mathrm{~cm}^{-3}$ is possible which is considerably higher than a standard pulsed DC plasma which shows a typical ion density of $1 \times 10^{10} \mathrm{~cm}^{-3}$. It is also possible to achieve a conformal coating which exhibits low stress allowing thick films to be deposited in the region of $70 \mu \mathrm{m}[11]$.

\section{RESULTS \& DISCUSSION}

Multilayer DLC coatings deposited using the described technique were analyzed by various methods to depict the range of properties obtained from the resulting films. Specifically the hardness, scratch adhesion, corrosion, thickness and erosion properties of the coating are described herein.

\section{Film and Layer Thickness}

The technique used for determining the thickness is the Calotest system which is manufactured by CSM Instruments [12]. Typically a tungsten carbide ball of a known diameter $(\sim 20 \mathrm{~mm})$ is used with diamond slurry to wear through the film surface until the substrate has been reached by the ball. The film is then put under an optical microscope where geometry is used to measure the coating thickness by comparing the relative diameter of the exposed surface layers to the known diameter of the ball.

Figure 4 shows an optical microscopy image of a Calotest crater in a typical multilayer DLC deposition on a 2"diameter by 12" long 304 grade stainless steel tube. Using a silicon and hydrocarbon precursor, an initial layer of $\mathrm{SiC}$ is deposited upon the bare stainless steel substrate. This SiC adhesion layer is used to achieve a good chemical bond with the substrate which then allows a thick stack of DLC dominant films to be deposited on top of the adhesion layer. In this case, a high silicon doped DLC layer, 
DLC cap layer, low Si doped DLC layer and a final DLC cap layer are deposited on top of the adhesion layer to make up the full DLC based film [8]. The structure shown in Figure 3 has a total thickness of $\sim 19 \mu \mathrm{m}$.

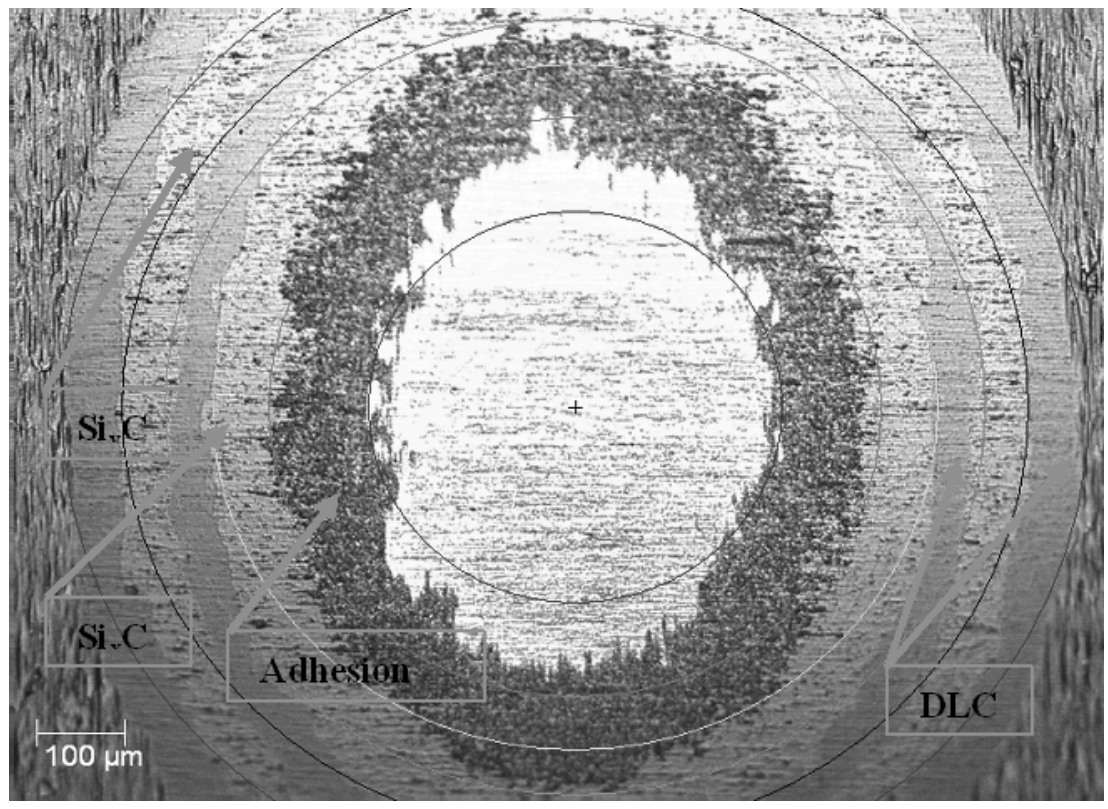

Figure 4: Image from optical microscope of film after calotest [8]

Figure 5 shows a SEM cross-section of similar multilayer coating structure with a total thickness of $40 \mu \mathrm{m}$ deposited by the PECVD. The total deposition time to grow such a structure was 100mins. In the SEM cameo image, the color is related to the EDX data, Areas that are warmer colors have higher average Z-number compositions. The brightest (warmest) region in the image is of the steel substrate. The bright green areas are the $\mathrm{Si}_{\mathrm{x}} \mathrm{C}_{\mathrm{y}}$, and the darkest regions are pure $\mathrm{C}$. There are layers of intermediate warmth, and those are the SiC/C mixed layer. The image shows the excellent step coverage obtained over defects on the steel pipe.

The described multilayer coating is deposited at a temperature of $\sim 200^{\circ} \mathrm{C}$ and when the coating relaxes back to room temperature, there is no sign of any cracking or breaking up of the film. The multilayer structure helps to reduce stress, improve corrosion and prevent crack propagation. For a single layer of DLC thick film of $20 \mu \mathrm{m}$ on SS304 substrate, as the coating cools back down to room temperature, the coating starts to break up and delaminate from the substrate. For most well-known deposition techniques, to get DLC to adhere to a metallic substrate, a film of a few $\mu \mathrm{m}$ thick is the limitation before degradation appears due to high residual stresses in the film $[13,14]$. DLC films of $20 \mu \mathrm{m}$ and above are extremely difficult to deposit but this technique can easily do this and also, a coating up to $70 \mu \mathrm{m}$ has been deposited using this technique with various hydrocarbon precursor combinations. 


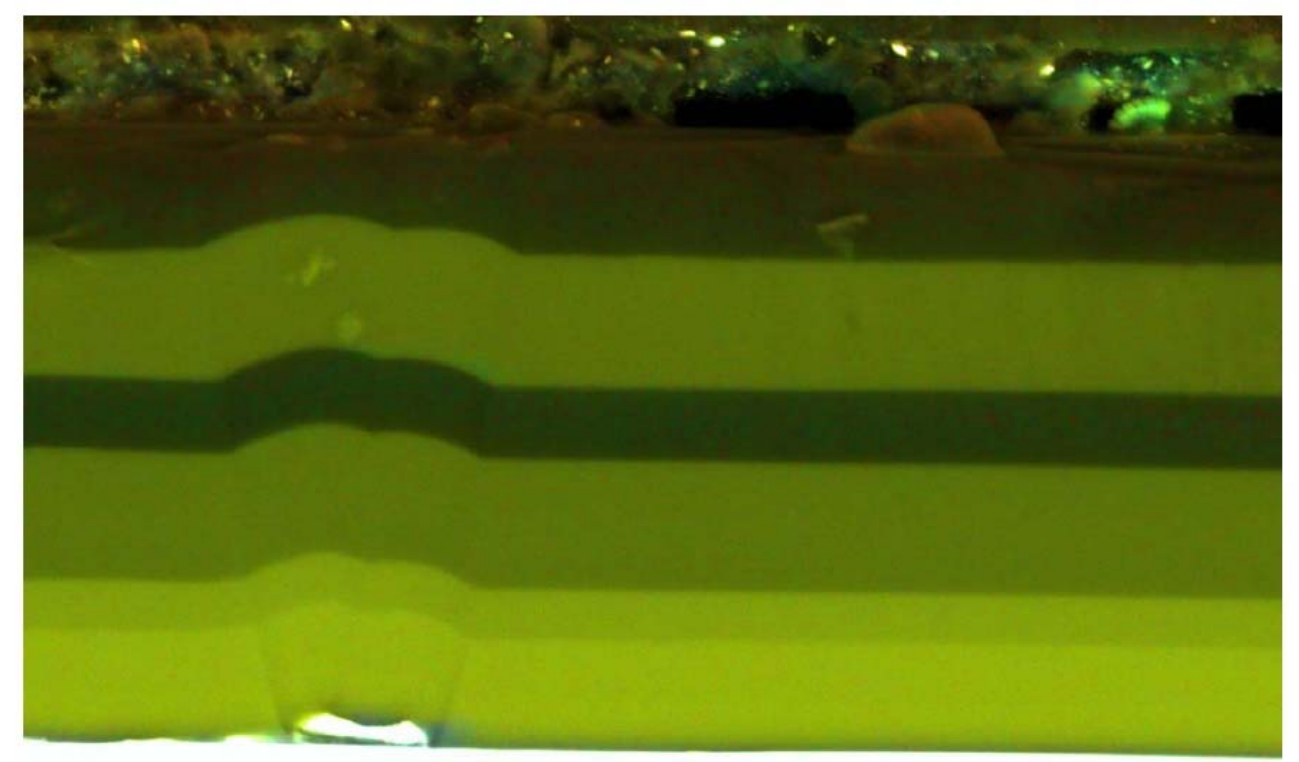

Figure 5: SEM cross-section of $\sim 40 \mu \mathrm{m}$ film on SS304 substrate

\subsection{Fracture behaviour and Erosion}

The coating fracture behaviour under a Vickers indenter load is shown in Figure 6 [8].



Figure 6: (a) Top view of $5 \mathrm{~N}$ indent and (b) SEM cross-section of indent after FIB [8]

Figure 6(a) shows a top view SEM image of a $5 \mathrm{~N}$ indent and 6(b) is a cross section of the indent area after FIB. The above indent is formed by an initial plastic deformation of the substrate. Cracks form within the coating at some initial load and when increasing the load to its maximum, crack growth takes place. Upon unloading of the tip, propagation of cracks can be seen at stress concentration points due to sharp indenter edges and finally delamination occurs within the layered structure. Most importantly, the cross-sectional image shows the benefits of a layered structure where deflection points are present within the structure for any crack propagation [8].

Multilayered films provide increased protection from erosion, additionally a harder substrate and thicker films provide enhanced erosion protection as shown in Fig 7. 


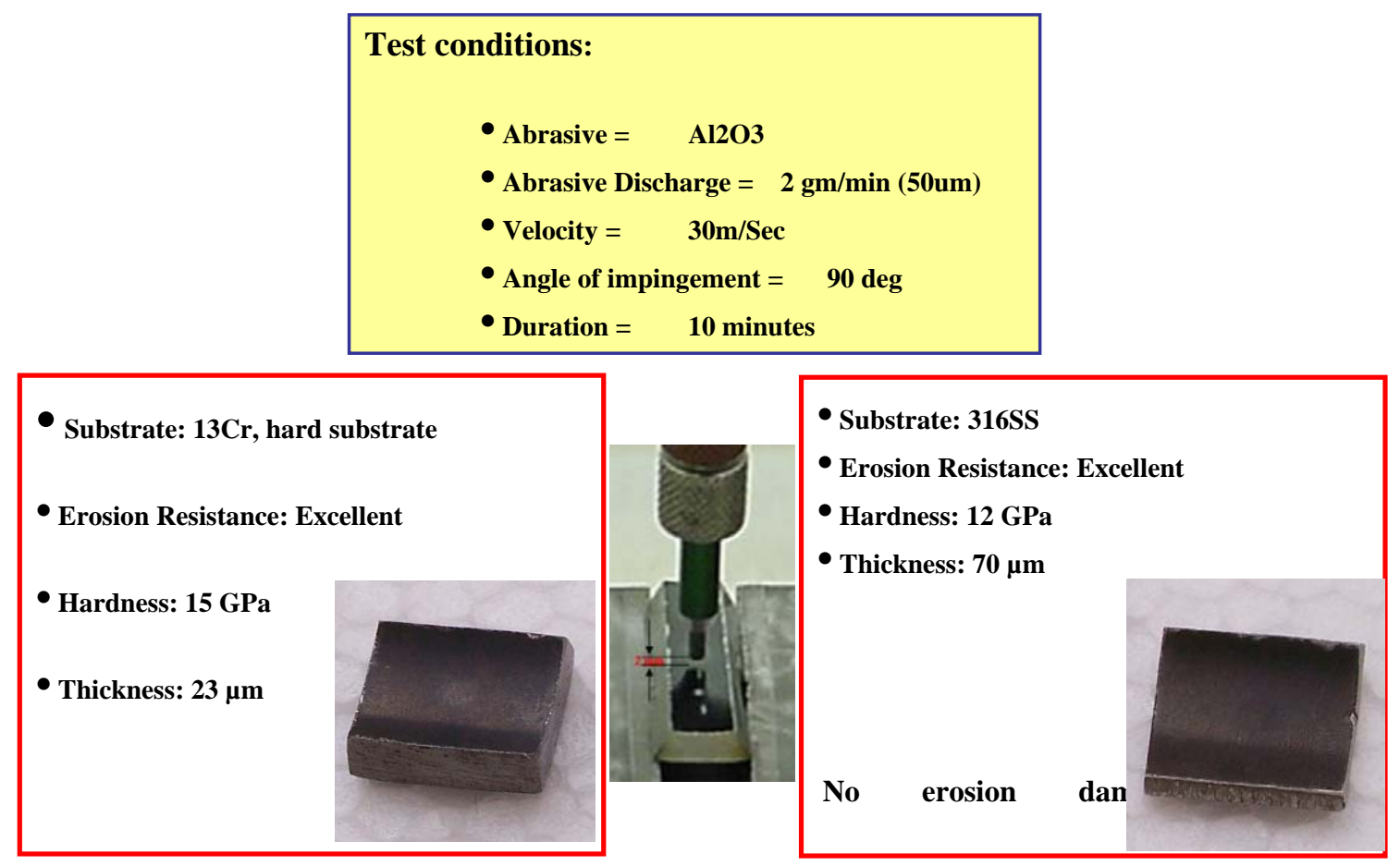

Figure 7: SEM cross-section of $\sim 40 \mu \mathrm{m}$ film on SS304 substrate

Here a gas jet erosion test is used to fire $50 \mu$ m diameter $\mathrm{Al}_{2} \mathrm{O}_{3}$ particles onto the surface of the coating at normal incidence for $10 \mathrm{mins}$. The coating is then removed from the rig and inspected for potential damage. On the left side of Figure 7 is a multilayer coating deposited on a $13 \mathrm{Cr}$ substrate where the hardness of the coating is $15 \mathrm{GPa}$ and total coating thickness of $23 \mu \mathrm{m}$. As can be seen, there is a slight colour change to the surface of the coating but no evidence of the abrasive breaking through the surface. Also shown in Figure 7 is another multilayer coating which is deposited on 316 stainless steel. This coating has a hardness of $12 \mathrm{GPa}$ and total thickness of $70 \mu \mathrm{m}$. This coating is also showing no penetration of the surface with this test. This clearly shows that the coating has excellent erosion resistant properties and for various substrate materials and film properties.

\subsection{Hardness/Wear}

When depositing films with the objective of them having high corrosion and erosion resistant properties, then the hardness and wear testing of these films is necessary. Figure 8 shows general properties for hardness and wear that are found from these DLC films.

\begin{tabular}{|l|l|l|l|l|l|l|}
\hline $\begin{array}{l}\text { Film } \\
\text { thickness } \\
\text { (um) }\end{array}$ & \multicolumn{2}{|l|}{$\begin{array}{l}\text { Young's Modulus } \\
\text { (GPa) }\end{array}$} & $\begin{array}{l}\text { Hardness } \\
\text { (GPa) }\end{array}$ & COF & $\begin{array}{l}\text { Wear Coefficient } \\
\mathrm{mm}^{3} / \mathrm{Nm}\end{array}$ \\
\cline { 2 - 5 } & DLC- & Substrate & DLC- & Substrate & DLC- & DLC-Si \\
\hline
\end{tabular}




\begin{tabular}{|l|l|l|l|l|l|l|}
\hline & Si & & Si & & Si & \\
\hline 25 micron & 184 & 193 & 22.7 & 2 & 0.02 & $4.72 \mathrm{E}-07$ \\
\hline
\end{tabular}

Figure 8: Hardness and Wear properties for bare and coated substrate [4]

From Figure 8 it is clear that a layer of DLC-Si on bare steel substrate greatly increases the hardness as expected. For the DLC-Si, the COF measured by tribometry is extremely low. 'The wear coefficient is measured using a linear reciprocating wear tribometer per ASTM G133, typically a $5 \mathrm{~mm}$ WC ball is used with an applied load of $5 \mathrm{~N}$, the wear track volume can then be measured from an optical profiler. The wear coefficient $\mathrm{k}$ is defined as $\mathrm{k}=$ (volume of material removed (mm3) / (Load in Newtons) $\mathrm{x}$ (length of wear track in meters). The value of wear coefficient confirms the excellent mechanical and adhesion properties of the film.

A more detailed analysis of the wear resistance of the coating using different liquid based media, including media containing highly abrasive particles such as titanium oxide (paint) and silica (drill mud), has also been carried out. Figure 9 details information about the various media used and values for the COF and wear rate.

\begin{tabular}{|l|l|l|l|} 
Substrate & Media & COF & $\begin{array}{l}\text { WearCoefficient } \\
\mathrm{mm} 3 / \mathrm{Nm}\end{array}$ \\
\hline \multirow{3}{*}{1020 Carbon steel } & Drill Mud & 0.04 & $6.55 \mathrm{E}-07$ \\
\cline { 2 - 4 } & Crude Oil & 0.03 & $2.65 \mathrm{E}-07$ \\
\cline { 2 - 4 } & Latex Paint & 0.03 & $1.40 \mathrm{E}-07$ \\
\cline { 2 - 4 } 316 stainless steel & Tap water & 0.16 & $8.10 \mathrm{E}-07$ \\
& Drill Mud & 0.02 & $1.20 \mathrm{E}-06$ \\
\cline { 2 - 4 } & Crude Oil & 0.05 & $1.45 \mathrm{E}-07$ \\
\cline { 2 - 4 } & Latex Paint & 0.1 & $8.40 \mathrm{E}-07$ \\
& Tap water & 0.09 & $4.05 \mathrm{E}-07$ \\
\hline Uncoated Steel & Dry & $0.4-0.6$ & $>\mathrm{E}-04$ \\
\hline
\end{tabular}

Figure 9: Wear resistant properties using various liquid based media

As shown in Figure 9 various different media have been looked at where the choice of what liquids to use was down to relevant applications for this coating within industry. The differences between the uncoated and coated substrate is clearly evident. In all 
cases excellent COF and wear rate have been demonstrated which further confirms the excellent wear properties of the coating and also indicates how versatile the coating is to different environments which opens up a huge number of applications.

\subsection{Corrosion}

Some examples of the corrosion testing methods that were done to test the corrosive properties will be shown. Figure 10 shows a test which was done involving a bare $13 \mathrm{Cr}$ substrate and a coated $13 \mathrm{Cr}$ substrate.
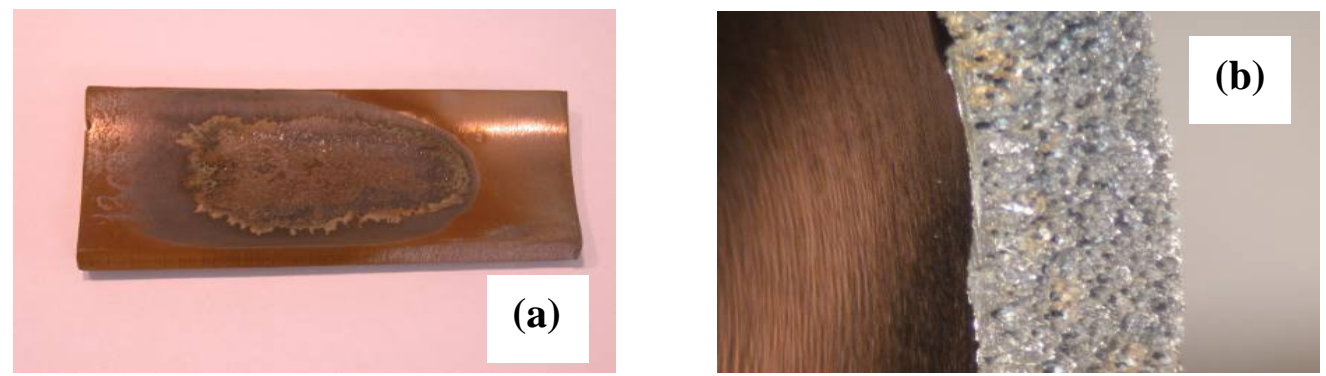

Figure 10: Bare 13Cr and coated 13Cr substrates after corrosion test [11]

Figure 10(a) shows the bare 13Cr substrate which was exposed to a drop of 15\% HCL at room temperature. It can be seen clearly how quickly and the extent of damage to the substrate when exposed to this solution. In contrast, Figure 10(b) is a coated $13 \mathrm{Cr}$ substrate which was submerged in HCL for 65hrs. What is evident from that test is that there is no damage to the film after being placed in such a highly corrosive environment and indicates good adhesion to the substrate and also the pinhole free nature of these films.

A more strenuous test of the film was conducted using a hot HCL solution with a coating on carbon steel (CS) 1020. Figure 11 shows the experimental set up for this test and Figure 12 shows the coating before and after this test and the test conditions. 




Pipe Cell fitted with condenser

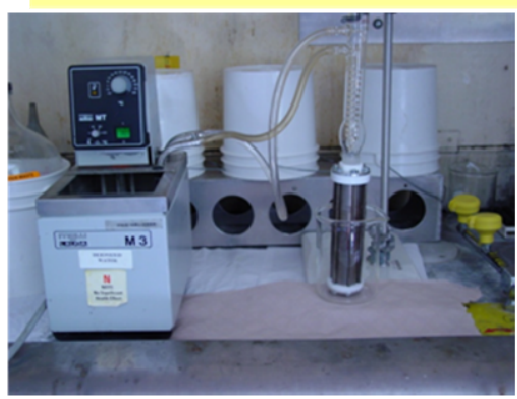

Assembled pipe Cell

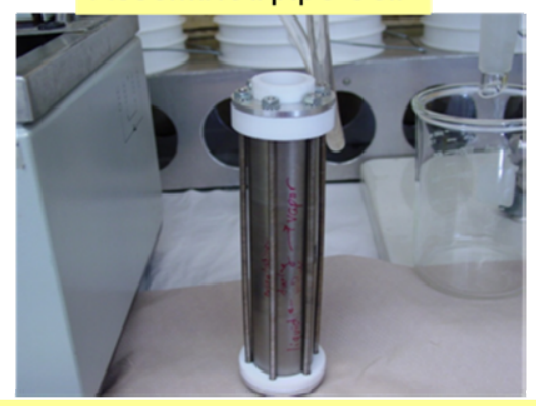

Full set up with TC and heat jacket



Figure 11: Experimental set up for hot HCL test
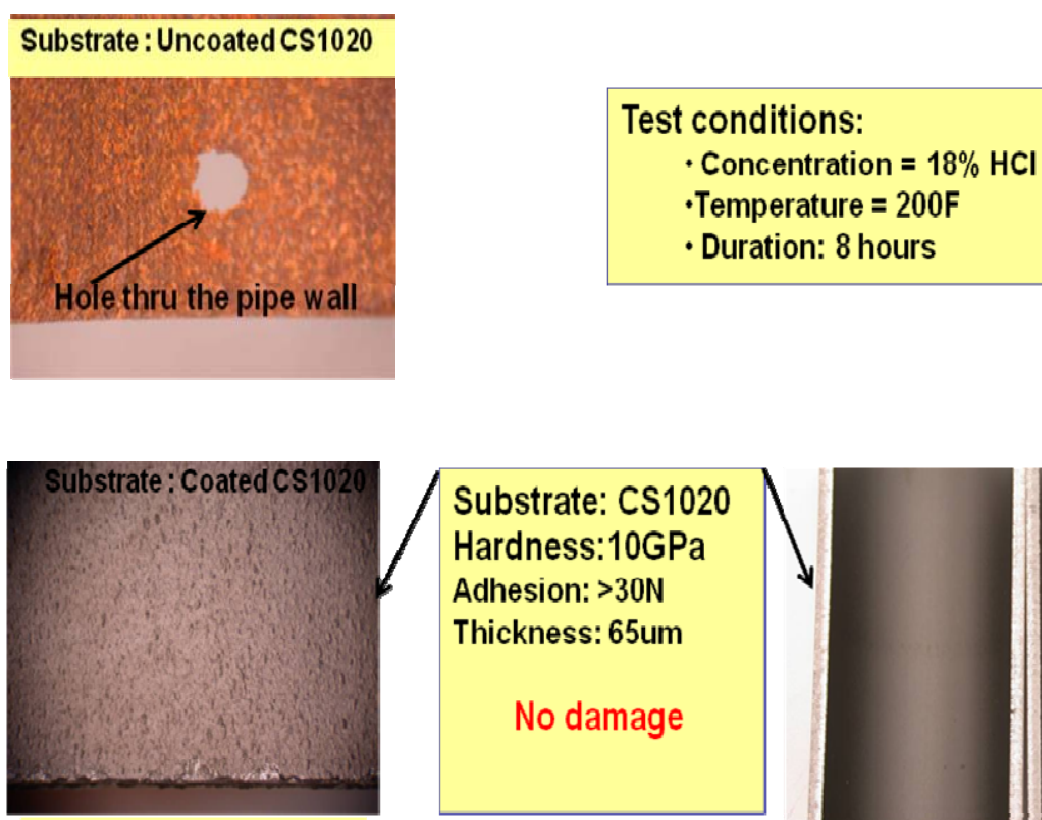

InnerArmor Protected
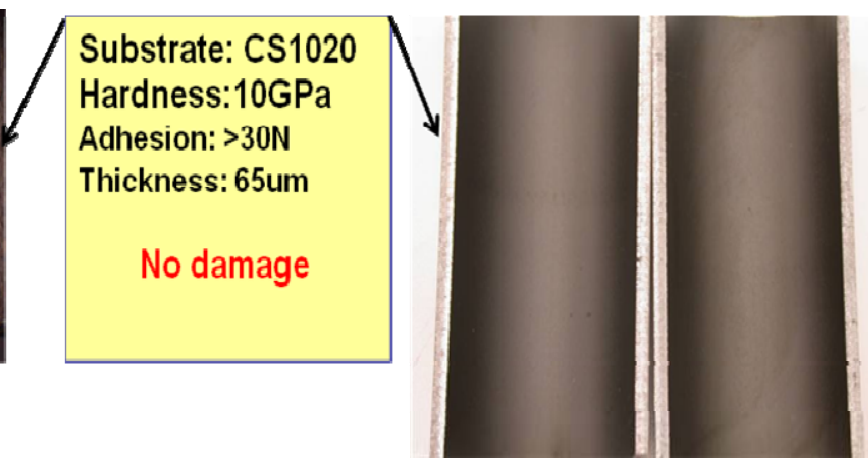

Figure 12: Coating on before and after hot HCL test and details of test conditions

From Figure 12, the degradation of the uncoated CS1020 substrate can clearly be seen under these conditions. Significantly, no damage is seen on the coating after this test. Although the film is not extremely hard, the very good scratch adhesion properties are being confirmed by this test and also the pinhole free nature of them. 
Another corrosion test which the coating has been subjected to was a sour autoclave. This is a three phase test (water based, organic and gas) which is done at high pressure. Figure 13 shows the film before and after a very aggressive test. This test is well known within the oil and gas industry as a good measure of how corrosion resistant the coating is.

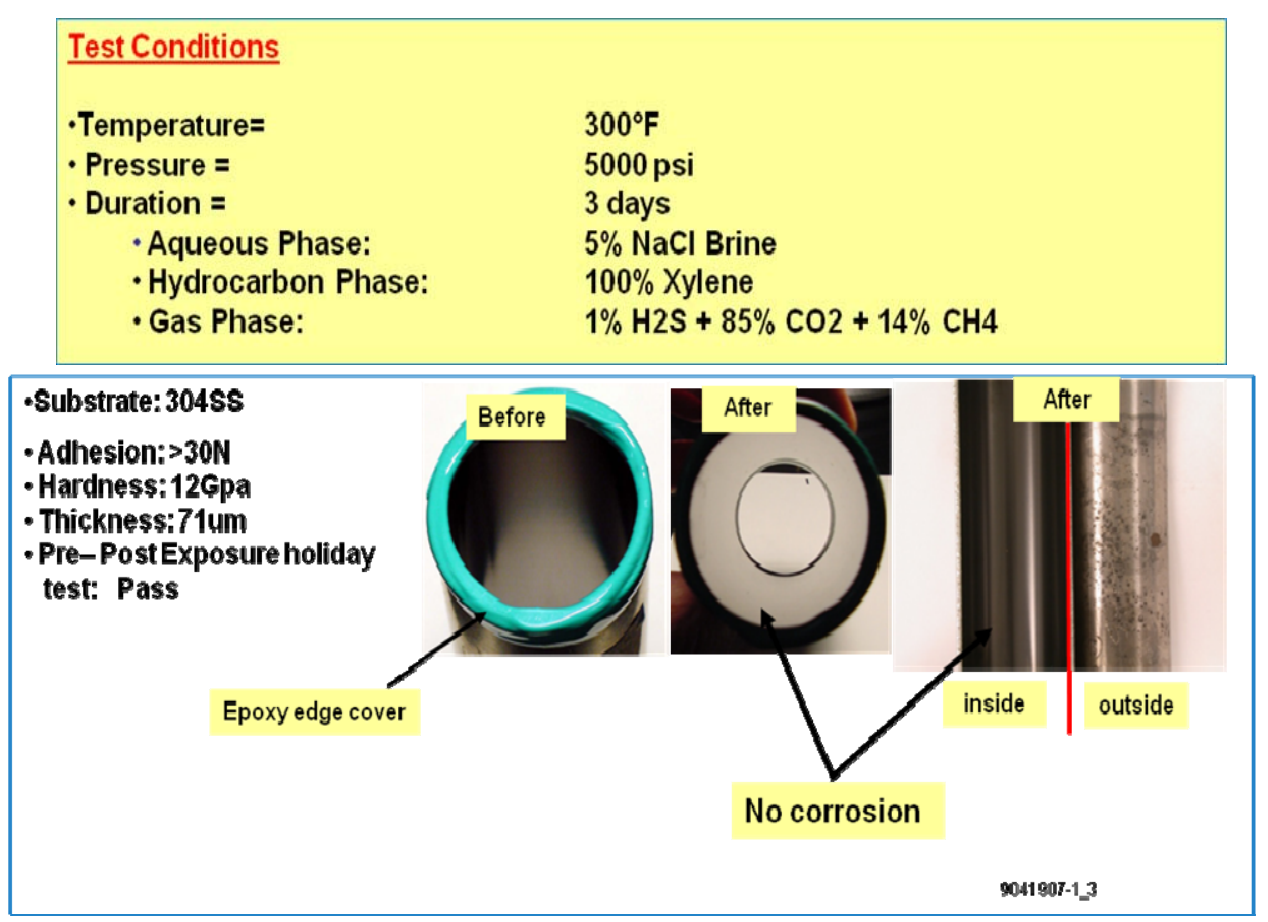

Figure 13: Coating before and after sour autoclave test and details of test conditions

Looking at the before and after test images, no delamination or penetration of the film surface is evident. Also, on the after image on the right of the figure, attack of the uncoated substrate can be seen and there has been no penetration of the corrosive chemicals to the interface of the film to substrate.

The corrosion results shown here are clearly demonstrating the extremely good corrosion resistant properties of the coating. Also by using some wide ranging corrosion tests, it opens up different applications where this coating can be used. Relevant applications will be discussed in the next section.

\section{APPLICATIONS}

The film properties achievable through the described PECVD process are quite unique, particularly on internal surfaces, and many applications can be envisioned where these properties could improve the useful service life or enhance the performance of industrial components. Potential applications include automotive, military, medical, aerospace, oil and gas and pulp and paper and many of these have been examined to various levels. Some examples of applications are for the military, the most obvious application is in gun barrels, in both a small and large scale. For the small scale, especially in sandy environments, there is a need to use arms without oils to stop 
jamming of guns. On the larger scale there is a need to replace $\mathrm{Cr}$ plating for large calibre guns used on ships, tanks and in the artillery [15].

For the medical industry there is a need for corrosion resistance on the inside of valves and tubes where good adhesion and smoothness is required. Also, implants for internal joints would need to be coated since these can corrode inside the human body and as DLC is chemically inert this would be advantageous for that [15].

For the pulp and paper industry, there is a need to replace expensive alloys which are used for corrosion resistance, with cheaper coated alloy steels. In the pulp mill, there are a large number of pipes and valves which carry corrosive material where a coating on the inside of these parts would increase the lifetime of the parts and be cost effective [15].

In geothermal systems, pipes are used to bring hot water from the ground and heat exchangers. Lower frictional losses means higher transfer rates and obviously in oil and gas you have offshore drilling and gas delivery systems which would benefit from this technology [15].

Further advancing the technology for extended geometry applications, such as transmission pipelines, will involve both further scaling of the process to accommodate the greater lengths and diameters associated with these applications as well as configuring the process to allow for on-site coating of seams, joints and repairs. Experiments and modelling to allow further scale-up are in progress and prototype insitu coating concepts are being explored.

\section{CONCLUSIONS}

A novel hollow cathode processing method has been described which can deposit thick DLC based multilayer coatings on the internal surfaces of cylindrical substrates. Details of the deposition system and technology have been shown with the actual part itself acting as the vacuum chamber and the cathode. In addition to the ability to coat internally, the process is capable of growing DLC based films to thickness in excess of $50 \mu \mathrm{m}$ at growth rates approaching $1 \mu \mathrm{m} /$ minute. These parameters are quite unusual in the current understanding of processing routes for DLC films and expand the scope and range of potential for commercial applications. A description of a multilayer DLC coating which has been deposited using this technology has been described and the most relevant testing procedures have been discussed in detail which demonstrate the unique combinations of high hardness, corrosion resistance and wear resistance, all with very good adhesion to the metallic substrate. Well adhered surface layers of this type offer a new way to protect internal surfaces and cavities including the inside of pipes. An extensive list of applications has been shown where corrosion and erosion problems have existed and it has been shown that many different industries can benefit greatly from this process. 


\section{REFERENCES}

1. H. Wesemeyer, H. Veltrop, US patent 5026466, "Method and device for coating cavities of objects,” June 1991

2. W.F. Henshaw, J.R. White, A. Niiler, US patent 4407712, "Hollow cathode discharge source of metal vapor,” October 1983

3. R.P. Fetherston, M.M. Shamim, J.R. Conrad, US patent 5988103, “Apparatus for plasma source ion implantation and deposition for cylindrical subtrates” Nov 1999

4. S. Eidelman, US Patent 6787194, "Method and apparatus for pulsed detonation coating of internal surfaces of small diameter tubes and the like” Sep 2004

5. S.D. Knowles, D.J. Senor, S.V. Forbes, R.N. Johnson, G.W. Hollenberg, US Patent 6866886, "Method of coating the interior surface of a hollow object with a diffusion coating” March 2005

6. J. Robertson, “Diamond-Like Amorphous carbon”, Materials Science and Engineering, R 37, pp. 129-281, 2002

7. Boardman et al., "Method and System for Coating Internal Surfaces of Prefabricated Process Piping in the Field", US Patent Application, Pub. No. US 2006/0011468 A1

8. T. Casserly, K. Boinapally, M. Oppus, D. Upadhyaya, B.Boardman, A. Tudhope, "Investigation of DLC-Si film deposited inside a 304SS pipe using a novel hollow cathode plasma immersion ion processing method", Proceedings of the Society of Vacuum Coaters Annual Technical Conference, 2007

9. T. Kalber, T. Jung, “A novel low-coat process for deposition of metallic and compound thin films on plastics”, Surface \& Coatings Technology 98 Jan 1998 11161120

10. L. Bardos, H. Barankova, "Hollow cathode PVD of nitride and oxide films at low substrate temperature”, Surface \& Coatings Technology 146-147 Sep-Oct 2001 463468

11. M. Gore, D. Lusk, A. Smith, "DLC coating of the internal surfaces of pipes using pulsed plasma PECVD techniques”, Plasmas Surfaces and Thin Films meeting, Institute of Physics June 2007

12. CSM Instruments SA, Rue de la Gare 4, Galileo Center, CH-2034 Peseux, Switzerland

13. D. Sheeja, B.K. Tay, L. Yin, S.P. Lau, "Low stress thick diamond-like carbon films prepared by filtered arc depsotion for tribological applications”, Surface \& Coatings Technology 1542002 289-293

14. O.R. Monteiro, J.Z. Ager III, D.H. Lee, R. Yu-Lo, K.C. Walter, M. Natasi, "Annealing of non hydrogenated amorphous carbon films prepared by filtered cathodic arc deposition”, Journal of Applied Physics 88 (5) 2000 2395-2399 
15. Sub-One Technology Technical Report, 2004 